\title{
Cairon. Vivre et mourir au Néolithique, la Pierre Tourneresse en Calvados
}

Nicolas Mathieu

\section{(2) OpenEdition \\ 12 Journals}

Édition électronique

URL : https://journals.openedition.org/rao/1864

DOI : $10.4000 /$ rao. 1864

ISBN : 978-2-7535-2790-4

ISSN : $1775-3732$

Éditeur

Presses universitaires de Rennes

Édition imprimée

Date de publication : 30 décembre 2012

Pagination : 319

ISBN : 978-2-7535-2641-9

ISSN : 0767-709X

\section{Référence électronique}

Nicolas Mathieu, «Cairon. Vivre et mourir au Néolithique, la Pierre Tourneresse en Calvados », Revue archéologique de l'Ouest [En ligne], 29 | 2012, mis en ligne le 30 décembre 2012, consulté le 22 juillet 2021. URL : http://journals.openedition.org/rao/1864 ; DOI : https://doi.org/10.4000/rao.1864

Ce document a été généré automatiquement le 22 juillet 2021.

@ Presses universitaires de Rennes 


\title{
Cairon. Vivre et mourir au Néolithique, la Pierre Tourneresse en Calvados
}

\author{
Nicolas Mathieu
}

\section{RÉFÉRENCE}

Ghesquière E., Marcigny C. (dir.), 2011 - Cairon. Vivre et mourir au Néolithique, la Pierre Tourneresse en Calvados, Rennes, PUR (coll. Archéologie et Culture), 200 p. (ISBN 978-2-7535-1438-6, $24 €)$.

1 Situé à une quinzaine de kilomètres au nord de Caen, un peu plus d'une dizaine de la côte, entre le village de Cairon et celui de Cairon-le-Vieux, sur une terrasse en rive droite dans le vallon du Vey, le dolmen de la "Pierre Tourneresse" ou "Pierre Tournerisse » a été l'objet de l'attention des érudits depuis la seconde moitié du xixe siècle au moins, si l'on en juge par un article de 1867 qui semble le plus ancien. Inscrit à l'Inventaire supplémentaire des Monuments historiques depuis 1954, il était réduit déjà à un amoncellement de dalles et de blocs consécutif à un abandon ancien après effondrement de sa couverture, la tombée dans l'oubli et les labours alentours qui ont contribué à accélérer les destructions et la remontée de blocs. À la fin du xxe siècle, le site ressemblait à une butte d'un mètre cinquante de haut et environ trente mètres de diamètre d'où dépassaient une grande dalle de calcaire et quelques blocs plus petits. La croissance urbaine de l'agglomération caennaise s'est manifestée par la construction d'un lotissement dans les années 1980, à moins d'une cinquantaine de mètres du site sans la moindre intervention archéologique préalable. Des prospections autour des vestiges dans le cadre de l'«Inventaire régional des monuments mégalithiques de Basse-Normandie » et un sondage destiné à évaluer le potentiel archéologique du site ont permis de l'identifier comme le vestige d'un cairn et ont favorisé en conséquence l'acquisition, par la municipalité de Cairon, du terrain ainsi qu'une première campagne 
de fouilles en 1996 dont les résultats prometteurs ont abouti à une autorisation de fouilles sur trois ans, de 1997 à 1999.

E. Ghesquière et C. Marcigny livrent dans cet ouvrage de belle facture (dans la collection maintenant bien connue et élégante « Archéologie et culture » dont le format permet une illustration lisible avec un cahier de planches hors texte en couleur) préparé avec la collaboration de quinze spécialistes - majoritairement archéologues (en archéozoologie, céramologie, anthracologie, carpologie, palynologie, pétrographie, etc.) mais aussi un anthropologue et un géographe - le résultat des fouilles et de leurs conséquences exemplaires, à savoir la mise en valeur du site. En effet, le parti a été pris, vu l'état de délabrement du cairn au moment des fouilles, de procéder à une fouille totale certes destructrice mais seule à même de permettre de répondre au plus grand nombre possible de questions, notamment architecturales - conception du cairn, éventuel tracé de son emprise, étapes de sa réalisation, de sa vie - puis à un remontage partiel sur une cinquantaine de centimètres de haut selon la règle actuelle de la réversibilité des restitutions dans le but pédagogique de rendre compréhensible au public le monument. Le cairn de Cairon est ainsi l'un des deux seuls (avec celui de Colombiers-sur-Seulles) monuments du Néolithique moyen présentés au public dans le Calvados.

3 La fouille totale a permis de lire les données paléo-écologiques du site, notamment de définir deux types de sol piégés, sous le monument d'une part et dans les éboulis d'autre part - que les auteurs appellent « vieux sol »- grâce au mobilier archéologique qu'ils contenaient (mobilier lithique, céramique, osseux par exemple) et qui fait l'objet de l'essentiel des présentations et analyses détaillées de la seconde partie du livre. Cette fouille complète a permis de mettre au jour une centaine de «structures archéologiques» au nombre desquelles une soixantaine de trous de poteau. Ces découvertes sont intéressantes chronologiquement et du point de vue régional. Elles témoignent d'un habitat datable du Néolithique moyen (seconde moitié de cette période 1: entre 4500 et 4350) qui a comporté un bâtiment, des palissades, de nombreux foyers et fosses et probablement un espace cultuel. Il n'est pas impossible qu'il y ait eu plusieurs phases de construction. À Cairon, il faut noter le faible intervalle entre l'occupation domestique et l'utilisation funéraire du lieu avec la construction du cairn: de l'ordre de quelques générations seulement. La comparaison avec d'autres sites normands conduit les auteurs à beaucoup de prudence dans l'interprétation chronologique de cette succession rapide et dans sa signification. Ponctuellement, on ne peut exclure une certaine "proximité cognitive» (p. 183) qui ne signifie pas que le cairn aurait été un lieu de mémoire ni encore moins un lieu à vocation cultuelle ou au sens magique. Que plusieurs autres sites normands aient associé successivement occupation humaine des vivants et cairn pourrait aussi bien s'expliquer par une raison pratique: l'existence d'un espace déjà débarrassé de la végétation gênante pour l'installation d'un cairn.

4 Le dolmen proprement dit est un cairn qui complète les enseignements sur les tombes à couloir déjà connues dans la plaine de Caen et leur étude architecturale puisque comme plus d'une vingtaine d'autres, c'est un cairn circulaire - avec une ou deux chambres circulaires - caractéristique du mégalithisme dans cette zone. Comme eux, il appartient à la phase culturelle du Néolithique moyen 2 et aux premières architectures mégalithiques monumentales de Normandie. 
5 Avec un parement de 25 mètres de diamètre, le cairn de Cairon est l'un des plus grands de Normandie. Il comporte deux chambres funéraires. La première est accessible par un couloir d'une dizaine de mètres de long. Le caveau, au plan quadrangulaire $(5 \times 3 \mathrm{~m})$ attesté aussi dans le Morbihan (Kermarker, à La Trinité-sur-Mer, et Rondossec, à Plouharnel) a livré les restes d'au moins une dizaine d'individus et les vestiges de deux ou peut-être trois coupes à socle quadrangulaire probablement associées à ces individus. De taille plus réduite, la seconde chambre, ouverte dans l'axe de la première sur le bord opposé, a une forme en bouteille sans distinction nette entre un couloir et une chambre. Elle a abrité un seul individu - ce qui est rare en Normandie dans les tombes à couloir non adventice, c'est-à-dire qui n'ont pas été construites dans une seconde phase - de quatre ans environ (datation par la dentition) qui reposait sur un dallage sommaire butant sur une banquette parementée. L'individu portait peut-être une parure. En tout cas a été retrouvé un petit pendentif avec un trou circulaire, issu d'un cerf (une (?) « crache» (?) de cerf : p. 48-49. On cherche aussi vainement la perle !). Les datations permises par l'analyse de l'ensemble du mobilier situent le fonctionnement de la première chambre vers 3900-3700 et un effondrement vers 3700-3500. Pour la chambre 2, l'utilisation date de la charnière IVe-IIIe millénaire avant notre ère. Les os de faune piégés dans le cairn attestent une fréquentation assidue du lieu à la fin de la première moitié du IVe millénaire avant notre ère sans qu'il soit possible de déterminer la nature de la fréquentation et de l'utilisation: banquet ou activité domestique.

6 Ce livre est finalement exemplaire à plusieurs titres. En premier lieu, par la qualité matérielle et la richesse de la documentation mise à la disposition des lecteurs; en deuxième lieu par la clarté du propos, la fluidité de la langue qui évite jargon et impropriétés - ce qui n'est pas toujours le cas dans la communauté des archéologues; en troisième lieu, par la préservation de la précision et de la rigueur scientifique de l'analyse. Ainsi, l'ouvrage doit trouver un public plus large que le seul milieu des chercheurs, des universitaires ou des étudiants préhistoriens. Historiens des mondes anciens soucieux d'insérer leur questionnement et leur curiosité dans la longue durée et dans la comparaison, érudits locaux ou régionaux, curieux et amateurs, non seulement bas-normands mais d'horizons plus vastes ou éloignés devraient y trouver leur compte.

\section{AUTEURS}

\section{NICOLAS MATHIEU}

Professeur d'histoire romaine - université Pierre-Mendès-France, Grenoble 2 